8th Alexander Friedmann International Seminar

on Gravitation and Cosmology

International Journal of Modern Physics: Conference Series

Vol. 3 (2011) 541-554

(C) World Scientific Publishing Company

DOI: 10.1142/S201019451100105X

\title{
WHAT IS CREDIBLE AND WHAT IS INCREDIBLE IN THE MEASUREMENTS OF THE CASIMIR FORCE
}

\author{
G. L. KLIMCHITSKAYA ${ }^{1,2}$ and V. M. MOSTEPANENKO ${ }^{1,3}$ \\ ${ }^{1}$ Department of Physics, Federal University of Paraíba, C.P. 5008, CEP 58059-900, \\ João Pessoa, Pb-Brazil \\ ${ }^{2}$ North-West Technical University, Millionnaya Street 5, St.Petersburg, 191065, Russia \\ ${ }^{3}$ Noncommercial Partnership "Scientific Instruments", Tverskaya Street 11, \\ Moscow, 103905, Russia
}

Received 2 June 2011

\begin{abstract}
We comment on progress in measurements of the Casimir force and discuss what is the actual reliability of different experiments. In this connection a more rigorous approach to the usage of such concepts as accuracy, precision, and measure of agreement between experiment and theory, is presented. We demonstrate that all measurements of the Casimir force employing spherical lenses with centimeter-size curvature radii are fundamentally flawed due to the presence of bubbles and pits on their surfaces. The commonly used formulation of the proximity force approximation is shown to be inapplicable for centimeter-size lenses. New expressions for the Casimir force are derived taking into account surface imperfections. Uncontrollable deviations of the Casimir force from the values predicted using the assumption of perfect sphericity vary by a few tens of percent within the separation region from 1 to $3 \mu \mathrm{m}$. This makes impractical further use of centimeter-size lenses in experiments on measuring the Casimir force.
\end{abstract}

Keywords: Casimir force; precise measurements.

PACS numbers: $12.20 .-\mathrm{m}, 42.50 . \mathrm{Ct}, 78.20 . \mathrm{Ci}$

\section{Introduction}

Experiments on measuring the Casimir force attracted considerable interest and raised heated debate. The paper ${ }^{1}$ reviews some recent work on this subject with a reservation that only "credible" experiments are considered. As incredible, the experiments which have claimed " $1 \%$ or better agreement" are meant with a generic reference to review. ${ }^{2}$ In different places, these unspecified incredible experiments are characterized" as " $1 \%$ level work", experiments "that claim $1 \%$ accuracy", and "experiments claiming $1 \%$ precision". In the first part of Ref. 1 the author provides arguments why it is unclear to him "what these experiments really mean". The second part of Ref. 1 is largely devoted to different aspects of author's own work employing spherical lenses of more than $10 \mathrm{~cm}$ curvature radius.

Below we demonstrate that the author's arguments against what he calls a " $1 \%$ level work" are based on incorrect or incomplete information. With respect to 
measurements of the Casimir force using lenses of centimeter-size curvature radii, we show that they are fundamentally flawed. According to our calculations, experiments of this type may lead to unpredictable results, due to unavoidable deviations from a spherical shape of mechanically polished and ground surfaces.

The paper is organized as follows. In Sec. 2 we explain what is incorrect in the argumentation $^{1}$ against precise experiments on measuring the Casimir force. We consider a relationship among the concepts of accuracy, precision and measure of agreement with theory, discuss total experimental error, its constituents, and rules of their combination, explain the misjudgement of constraints on long-range forces made. ${ }^{1}$ In Sec. 3 it is shown that all measurements of the Casimir force employing centimeter-size spherical lenses are fundamentally flawed. We discuss both the electrostatic calibrations and the measurement of the Casimir force. We demonstrate that commonly used simplified form of the proximity force approximation (PFA) is inapplicable in the presence of standard imperfections on the optical surfaces (bubbles and pits) and derive new expressions for the Casimir force valid in the presence of these imperfections. In Sec. 4 some further objectionable features of Ref. 1 are discussed. Sec. 5 contains our conclusions and discussion.

\section{What Is Incorrect in the Arguments Against Precise Experiments}

\subsection{Confusion between accuracy, precision and measure of agreement with theory}

The present state of the art in experiments on measuring the Casimir force is reflected in the review. ${ }^{2}$ Some results in this review do not necessarily coincide with respective formulations in original publications because several experiments were later reanalyzed using more reliable methods of data processing. At the moment only these updated results ${ }^{2}$ should be used in all discussions. In so doing, each experiment on measuring the Casimir force is characterized by a total experimental error, total theoretical error, and measure of agreement between experiment and theory determined at some high (usually 95\%) confidence level. ${ }^{2}$ Therefore the above cited characterizations of precise experiments, ${ }^{1}$ which confuse $1 \%$ agreement, $1 \%$ accuracy and $1 \%$ precision are completely misleading. According to Ref. 2, the best measure of agreement between the Casimir pressure measured in the most precise experiment ${ }^{3}$ using a micromachined oscillator and theory is equal to $1.8 \%$ at the separation $a=400 \mathrm{~nm}$ between the test bodies. As to the best measure of agreement between the measured Casimir force in sphere-plate geometry and theory in the most precise experiment ${ }^{4}$ using an atomic force microscope, it is equal ${ }^{5}$ to $5.4 \%$ at separations around $a=80 \mathrm{~nm}$. Hence the " $1 \%$ or better agreement" is an incorrect information.

Of even greater concern is the mention" of experiments "that claim $1 \%$ accuracy". Actually, there are no such experiments. ${ }^{2}$ What's more, measuring of accuracy in percents ${ }^{1}$ is in contradiction with the rigorous understanding of this concept. 
According to the International vocabulary of metrology ${ }^{6}$ produced by the Joint Committee for Guides in Metrology, measurement accuracy is the closeness of agreement between a measured quantity value and a true quantity value. It is underlined ${ }^{6}$ that the concept "measurement accuracy" is not a quantity and is not given a numerical value. This interpretation is well founded because a true quantity value is in principle unknown. A measurement is said to be more accurate when it offers a smaller measurement error. Thus, it is meaningless to speak about $1 \%$ or any other numerical degree of accuracy, and attribute it to some unspecified experiments. ${ }^{1}$

Another concept used $^{1}$ in the same context is the concept of precision. Measurement precision is the closeness of agreement between measured quantity values obtained by replicate measurements on the same or similar objects under specified conditions. ${ }^{6}$ Precision is expressed numerically by measures of imprecision. ${ }^{6}$ Specifically, the experimental errors can be used as such measures. In the experiment ${ }^{3}$ the highest measurement precision is achieved at the separation $a=162 \mathrm{~nm}$ where the total relative experimental error is equal to ${ }^{2}$

$$
\delta_{t} \Pi^{\text {expt }}=\frac{\Delta_{t} \Pi^{\text {expt }}}{\left|\Pi^{\text {expt }}\right|}=0.19 \%,
$$

where $\Pi^{\text {expt }}$ is the measured value of the physical quantity $\Pi$ (the Casimir pressure between two parallel plates), and $\Delta_{t} \Pi^{\text {expt }}$ is the total absolute experimental error. In the experiment ${ }^{4}$ the highest precision is achieved at $63 \mathrm{~nm}$ and corresponds to the total relative experimental error ${ }^{2} \delta_{t} \Pi^{\text {expt }}=1.5 \%$, where $\Pi^{\text {expt }}$ is the measured Casimir force between a sphere and a plate. Although it is recognized ${ }^{1}$ that "There is a tendency among workers in this field to confuse precision with accuracy, of which I am guilty myself," the definitions presented ${ }^{1}$ continue to be unrelated to the rigorous formulations. ${ }^{6}$ Specifically, precision relates not to the number of significant figures provided by a measurement device, as erroneously stated, ${ }^{1}$ but to a closeness between measured quantity values in replicate measurements (the same voltmeter, for instance, can be used in different experiments leading to different precisions).

\subsection{Total experimental error and its constituents}

It is argued" that "to obtain a given experimental accuracy, say $1 \%$, requires that the calibrations and force measurements must be done to much better than $1 \%$ accuracy..." However, the suggested arguments that the latter is yet not possible contain several incorrect statements. Before we indicate each of the specific mistakes made, let us emphasize that the word "accuracy" used ${ }^{1}$ must be replaced with the word "precision" because, as explained above, accuracy is not given a numerical value and there are no experiments claiming a $1 \%$ accuracy.

It is right that if, for instance, the total experimental error is equal to $1 \%$ all calibration errors must be smaller accordingly depending on their size and number. The list of these errors ${ }^{1}$ is, however, again confusing by mixing experimental errors, theoretical errors and agreement between experiment and theory. Here, we illustrate 
what are the constituents of the lowest total experimental error $\delta_{t} \Pi^{\text {expt }}=0.19 \%$ at a separation $a=162 \mathrm{~nm}$ in the most precise experiment. ${ }^{3}$ The relative random error in the Casimir pressure at $a=162 \mathrm{~nm}$ is ${ }^{2} \delta_{r} \Pi^{\operatorname{expt}}=0.04 \%$. It is determined from the standard statistical procedure using Student distribution. ${ }^{7}$ The systematic error is caused by the errors in the measurement of the sphere radius, of the frequency shift, and of the proximity force approximation (which is a part of experimental procedure in the indirect measurement of the Casimir pressure). The resulting relative systematic error at a separation of $162 \mathrm{~nm}$ is $\delta_{s} \Pi^{\text {expt }}=0.19 \%$. It is claimed ${ }^{1}$ that for achieving a $1 \%$ experimental accuracy (read precision) the sphere radius needs to be measured to $0.5 \%$ accuracy (precision), and that "the radius measurement is not discussed in sufficient details in any of papers..." This is, however, not so. The value of the sphere radius in the experiment ${ }^{3}$ was determined to be $R=151.2 \pm 0.2 \mu \mathrm{m}$ leading $^{2,3}$ to the relative error of only $0.13 \%$, i.e., smaller error than is demanded. ${ }^{1}$ In fact all the details for determination of sphere radius by means of electrostatic calibrations are provided..$^{3,8,9}$

The other sources of errors considered ${ }^{1}$ are unrelated to the experimental precision. Thus, the knowledge of the optical properties of the surfaces is not needed for the determination of precision. The discussion of errors in the Casimir force induced by the errors in absolute separations bears no relation to force and pressure measurements as well. The separation distance is an independent quantity and is measured with its own measurement error (in Ref. 3 the latter is equal to $0.6 \mathrm{~nm}$ ). Both these errors are important for the comparison between experiment and theory, but have nothing to do with the achieved experimental precision of force and pressure measurements. ${ }^{2}$

\subsection{Is it really uncertain how to combine different errors and uncertainties?}

As discussed above, the total experimental error results from the combination of random and systematic errors. In its turn, the systematic error has several constituents. According to Ref. 1, precision measurement experts still debate whether these errors and uncertainties can be added in quadrature or be simply added. Regarding this statement we suggest that the author ${ }^{1}$ was guided by outdated information. It is common knowledge that errors and uncertainties are random quantities and are characterized by some distributions. ${ }^{10}$ The composition law of several random quantities depends on the specific form of these distributions. In the measurements of the Casimir force it is usually supposed that all systematic errors in the form of systematic deviations (i.e., biases in a measurement which always make the measured value higher or lower than the true value) are already removed using some known process, i.e., through a calibration. The remaining systematic errors are the errors of a calibration device and have the meaning of the smallest fractional devision of the scale of the device. Such systematic errors are random quantities characterized by a uniform distribution (equal probability). The errors in an approximate theoretical 
formula used to convert a directly measured quantity into an indirectly measured one are also distributed uniformly. Then the resulting systematic error at a chosen confidence level $\beta$ is obtained from its constituents $\Delta_{s}^{(i)} \Pi^{\operatorname{expt}}(i=1,2, \ldots, J)$ using the following statistical rule ${ }^{10}$

$$
\Delta_{s} \Pi^{\text {expt }}=\min \left[\sum_{i=1}^{J} \Delta_{s}^{(i)} \Pi^{\text {expt }}, k_{\beta}^{(J)} \sqrt{\sum_{i=1}^{J}\left(\Delta_{s}^{(i)} \Pi^{\text {expt }}\right)^{2}}\right] .
$$

Here, $k_{\beta}^{(J)}$ is a tabulated coefficient. The above value of $\delta_{s} \Pi^{\text {expt }}=0.19 \%$ at $a=$ $162 \mathrm{~nm}$ in the experiment ${ }^{3}$ (see Sec. 2.2) was obtained ${ }^{2,5}$ using Eq. (2) with $J=3$, $\beta=0.95$, and $k_{0.95}^{(3)}=1.1$.

Contrary to Ref. 1, statistical rules for the combination of random and systematic errors have also been much studied. The random error is described by the normal or Student distribution. The resulting systematic error is described by a combination of uniform distributions. It can be shown that if the resulting systematic error is also assumed to be distributed uniformly, the total experimental error will be overestimated. Thus, this assumption is conservative and can be used safely. There are several methods in statistcs how to combine errors described by normal and uniform distributions. ${ }^{10} \mathrm{~A}$ widely used method puts

$\Delta_{t} \Pi^{\operatorname{expt}}=\Delta_{r} \Pi^{\operatorname{expt}}, \quad \Delta_{t} \Pi^{\text {expt }}=\Delta_{s} \Pi^{\operatorname{expt}}, \quad \Delta_{t} \Pi^{\operatorname{expt}}=q_{\beta}(r)\left[\Delta_{r} \Pi^{\text {expt }}+\Delta_{s} \Pi^{\text {expt }}\right]$

depending on what respective inequality is fulfiled for all $a$ over the entire measurement range $r(a)<0.8, r(a)>8$, or $0.8 \leq r(a) \leq 8$. Here, the quantity $r(a)$ is defined as $r(a)=\Delta_{s} \Pi^{\operatorname{expt}}(a) / s_{\bar{\Pi}}(a)$, where $s_{\bar{\Pi}}(a)$ is the variance of the mean of a quantity $\Pi^{\text {expt }}$. The coefficient $q_{\beta}(r)$ at a confidence level $\beta=0.95$ varies between 0.71 and 0.81 depending on the value of $r(a)$. Note that the value of the relative total error in the experiment ${ }^{3}$ at $a=162 \mathrm{~nm}$ was obtained using the second equality in (3). We emphasize that the dominance of the resulting systematic error over the random error within the entire measurement range achieved in the experiment ${ }^{3}$ is the distinguishing feature of precise experiments of a metrological quality.

From the above it is seen that precision measurement experts have gone far beyond debates whether uncertainties can be added in quadrature or simply added.

\subsection{Misjudgement of constraints on long-range forces following from the most precise Casimir experiment}

The measure of agreement between the Casimir pressures measured in the most precise experiment ${ }^{3}$ and calculated theoretically was used to obtain the strongest constraints on the parameters of long-range Yukawa-type forces in the interaction range of several tens of nanometers. ${ }^{3,5}$ In doing so the Yukawa pressure was calculated $^{3}$ by the application of the PFA. The paper ${ }^{1}$ informs the reader that the use of this approximation has been criticized. ${ }^{11}$ The conclusions ${ }^{11}$ that the PFA "only 
applies to a force that depends on the location of body surfaces" and "is not valid for the volume integral required for calculating the anomalous force" are repeated. ${ }^{1}$

This conclusion is, however, incorrect as is demonstrated in available literature overlooked by the author. ${ }^{1}$ Thus, it is shown ${ }^{12}$ that the PFA is applicable for the calculation of the Yukawa force under conditions that the separation $a$ and interaction range $\lambda$ are much smaller than the sphere radius $R$ and the plate thickness $D$. All these conditions are satisfied with a large safety margin in the experimental setup. ${ }^{3}$ The respective Yukawa pressure in the setup ${ }^{3}$ was calculated ${ }^{13}$ both exactly and using the PFA with coinciding results. The purported "corrections" to the calculation ${ }^{12}$ pointed out ${ }^{11}$ were shown to be invalid and based on a simple misunderstanding. ${ }^{13}$ What's more, one of the authors ${ }^{11}$ (R.O.) recognized ${ }^{14}$ that the issue raised in their paper "is not of practical concern for current experiments". Thus, the reliability of constraints on the parameters of Yukawa interactions obtained ${ }^{3}$ is beyond doubt.

\section{Why Casimir Force Measurements Using Centimeter-Size Spherical Lenses Are Fundamentally Flawed}

\subsection{Anomalies in electrostatic calibrations}

Observations of anomalous electrostatic forces in the lens-plate geometry for lenses of centimeter-size curvature radii is the subject of wide speculation. ${ }^{15-17}$ It was shown $^{18}$ that anomalous behavior of the electrostatic force can be explained due to deviations of the mechanically polished and ground surfaces of centimeter-size lenses from a perfect spherical form. The point is that the typical surface of a centimeter-size lens is characterized in terms of scratch and dig optical surface specification data. In particular, depending on the quality of lens used, bubbles and pits with a diameter varying from $30 \mu \mathrm{m}$ to $1.2 \mathrm{~mm}$ are allowed on the surface. ${ }^{19}$ There may be also scratches ${ }^{19}$ with a width varying from 3 to $120 \mu \mathrm{m}$. The problem of bubbles on the centimeter-size lens surface should not be reduced to the fact that lens curvature radius $R$ is determined with some error. The thickness of each bubble should of course be less than the absolute error in the measurement of lens curvature radius (for a lens ${ }^{16}$ with $R=15.10 \mathrm{~cm}$, for instance, $\Delta R=0.05 \mathrm{~cm}$ ). The crucial point is that curvature radii of bubbles can be orders of magnitude different, as compared to $R$. This allows one to suggest models leading to quite different ("anomalous") dependence of electrostatic force on separation in comparison with the case of perfect spherical surfaces. ${ }^{18}$

Paper $^{1}$ mentions the possibility that the anomalous electrostatic forces are due to simple geometrical effects without citing the source of this idea, but claims that this possibility "is credibly discarded" in Ref. 20. The author ${ }^{1}$ does not inform the reader that computations ${ }^{20}$ were repeated ${ }^{21}$ and shown to be not reproducible. Thus, there is no scientific objection against the possibility that anomalous electrostatic forces are due to deviations of mechanically polished and ground surfaces from perfect sphericity. Furthermore, some of the authors ${ }^{20}$ (D.A.R.D. and R.O.) 
recently recognized ${ }^{22}$ that local geometrical deformations of the surface can really lead to an anomalous electrostatic force not only in sphere-plate geometry, but for a cylindrical lens in close proximity to the plate as well. It was underlined ${ }^{22}$ that "this is certainly a crucial point to be taken into account in future experiments." This reference, however, is missing in the list of references. ${ }^{1}$ An extensive consideration ${ }^{1}$ of the electrostatic calibrations always assumes perfect sphericity of the lens surface.

Another misrepresented point directly relevant to electrostatic calibrations is the dependence of the contact potential on the separation distance. It is claimed ${ }^{1}$ that every paper on the Casimir effect "that has bothered measuring the contact potential as a function of distance has shown an apparent distance dependence of that potential". This is, however, not the case. Thus, the contact potential was carefully measured as a function of separation ${ }^{3}$ and found to be constant. The respective measurement data of the electrostatic calibrations are published. ${ }^{9,18}$ Constant contact potential was observed in all other experiments by R. S. Decca as well. ${ }^{2,5}$ Independent on separation contact potential was also reported in experiments by U. Mohideen. ${ }^{2,4,5,23-25}$ It is notable that all these experiments were performed in high vacuum with small spheres of order $100 \mu \mathrm{m}$ curvature radii.

\subsection{Influence of surface imperfections on the Casimir force for lenses of centimeter-size curvature radius}

The Casimir force is far more sensitive than the electrostatic force to the bubbles and pits that are unavoidably present on the mechanically polished and ground surface of any lens of centimeter-size curvature radius. The physical reason is that the Casimir force falls with the increase of separation distance more rapidly than the electric force. As a result, it is determined by smaller regions near the points of closest approach of the surfaces. If the local curvature radius on the lens surface near the point of closest approach to the plate is significantly different from the mean lens curvature radius $R$, the impact on the Casimir force can be tremendous. Below we demonstrate that just this happens due to the presence of bubbles and pits on a lens surface. For simplicity, we consider ideal metal surfaces. However, it is shown ${ }^{26}$ that all conclusions obtained are preserved for real bodies as well.

The Casimir force in sphere-plate geometry under the experimental conditions $a \ll R$ is usually calculated using the PFA. ${ }^{2,5}$ According to the most general formulation of the PFA, ${ }^{27}$ the unknown force between the elements of curved surfaces is approximately replaced with a known force per unit area of the plane surfaces (i.e., a pressure) at the respective separation multiplied by an area element. Applied to a spherical lens of thickness $D$ above a plane $z=0$, the PFA represents the force between them in the form

$$
F_{s p}(a, T)=\int_{\Sigma} d \sigma P(z, T)
$$

Here, $d \sigma$ is the element of plate area, $\Sigma$ is the projection of the lens onto the plate, $a$ is the shortest separation between them, $z=z(x, y)$ is the equation of a lens 
surface, and $P(z, T)$ is the pressure for two plane parallel plates at a separation $z$ at temperature $T$.

We choose the origin of a cylindrical coordinate system on the plane $z=0$ under the lens center. Then for a perfectly shaped spherical lens the coordinate $z$ of any point of its surface is given by

$$
z=R+a-\left(R^{2}-\rho^{2}\right)^{1 / 2}, \quad \rho^{2}=x^{2}+y^{2} .
$$

In this case (4) leads to

$$
F_{s p}^{\text {perf }}(a, T)=2 \pi \int_{0}^{\sqrt{2 R D-D^{2}}} \rho d \rho P(z, T)=2 \pi \int_{a}^{D+a}(R+a-z) P(z, T) d z .
$$

Keeping in mind that the Casimir pressure is expressed as $P(z, T)=$ $-\partial \mathcal{F}_{p p}(z, T) / \partial z$, where $\mathcal{F}_{p p}(z, T)$ is the free energy per unit area of parallel plates, and integrating by parts in (6), one arrives at

$$
\begin{aligned}
F_{s p}^{\text {perf }}(a, T)= & 2 \pi R \mathcal{F}_{p p}(a, T)-2 \pi(R-D) \mathcal{F}_{p p}(D+a, T) \\
& -2 \pi \int_{a}^{D+a} \mathcal{F}_{p p}(z, T) d z .
\end{aligned}
$$

We consider centimeter-size spherical lenses satisfying a condition $a \ll D$. For such lenses $\mathcal{F}_{p p}(D+a, T) \ll \mathcal{F}_{p p}(a, T)$. Because of this, one can neglect the second term on the right-hand side of (7) in comparison with the first. ${ }^{28}$ It can be also shown ${ }^{28,29}$ that the first term on the right-hand side of $(7)$ is in excess of the third by a factor of $R / a$. This allows one to neglect the third term and arrive to what is called the simplified formulation of the $\mathrm{PFA}^{28,29}$

$$
F_{s p}^{\text {perf }}(a, T) \approx 2 \pi R \mathcal{F}_{p p}(a, T)
$$

widely used for both spherical lenses and for spheres [note that for a semisphere the second term on the right-hand side of (7) is identically equal to zero].

For two parallel ideal metal plates spaced $z$ apart the Casimir free energy per unit area is given by ${ }^{5,30}$

$$
\mathcal{F}_{p p}(z, T)=\frac{k_{B} T}{\pi} \sum_{l=0}^{\infty} \int_{0}^{\infty} k_{\perp} d k_{\perp} \ln \left(1-e^{-2 z q_{l}}\right) .
$$

Here, $k_{B}$ is the Boltzmann constant, $k_{\perp}$ is the magnitude of the projection of the wave vector on the plates, $q_{l}^{2}=k_{\perp}^{2}+\xi_{l}^{2} / c^{2}, \xi_{l}=2 \pi k_{B} T l / \hbar$ with $l=0,1,2, \ldots$ are the Matsubara frequencies, and the primed summation sign means that the term with $l=0$ is multiplied by $1 / 2$. For the sake of convenience in computations, we rewrite Eq. (9) in terms of a dimensionless integration variable $y=2 a q_{l}$ and expand the logarithm in power series. Then, after performing integration and the summation with respect to $l$, the following result is obtained:

$$
\mathcal{F}_{p p}(z, T)=-\frac{k_{B} T}{4 \pi z^{2}}\left[\frac{\zeta(3)}{2}+\sum_{n=1}^{\infty} \frac{e^{-\tau_{z} n}}{n^{2}\left(1-e^{-\tau_{z} n}\right)}\left(\frac{1}{n}+\frac{\tau_{z}}{1-e^{-\tau_{z} n}}\right)\right]
$$




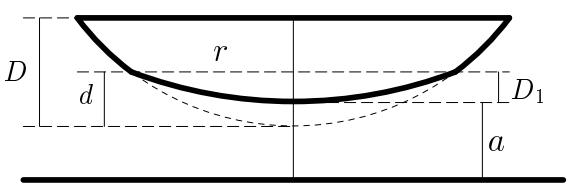

(a)

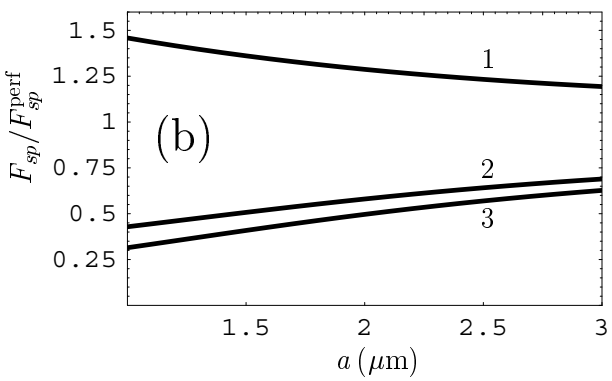

Fig. 1. (a) The configuration of a spherical lens with curvature radius $R$ possessing a surface imperfection at the point of closest approach to a plate. The bubble curvature radius is $R_{1}>R$. The relative sizes of the lens and imperfection are shown not to scale. (b) The normalized Casimir force acting between a sphere with surface imperfections of different types and a plate as a function of separation. Lines 1, 2, and 3 are for the surface inperfections shown in Figs. 1(a) and 2(a,b), respectively.

where $\zeta(x)$ is the Riemann zeta function, and $\tau_{z}=4 \pi z k_{B} T /(\hbar c)$. Note that the first contribution on the right-hand side of (10) coincides with the high temperature limit of the free energy. This is quite reasonable if to take into account that $\tau_{z}=2 \pi T / T_{\text {eff }}$, where the effective temperature is defined from $k_{B} T_{\text {eff }}=\hbar c /(2 z)$.

Now we are in a position to compute the Casimir force between real spherical lens of large curvature radius with bubbles and pits of different types and a plane plate. It is common to use the simplified formulation of the PFA (8) in sphere-plate geometry for both small spheres of about $100 \mu \mathrm{m}$ radii and large spherical lenses (see, for instance, Refs. 3, 4, 16, 31, 32). In doing so the role of bubbles and pits on the surface of lenses of centimeter-size curvature radii is simply disregarded. Equation (8), however, is not applicable for real lenses with large curvature radii because it assumes perfect spherical surface. For such lenses one should use a general formulation of the PFA in Eq. (4). To illustrate this fact, we perform calculations for three typical model imperfections on the spherical surface near the point of closest approach to the plate allowed by the optical surface specification data. ${ }^{19}$

As the first example, we consider a bubble of the curvature radius $R_{1}=25 \mathrm{~cm}$ which is larger than the curvature radius $R=15 \mathrm{~cm}$ of the lens used [see Fig. 1(a)]. The thickness of the spherical lens formed by the bubble is chosen to be $D_{1}=0.5 \mu \mathrm{m}$ (this is much less than typical absolute error $\Delta R=0.05 \mathrm{~cm}$ in the measurement of centimeter-size lenses curvature radius). The radius of the bubble is determined from $r^{2}=2 R_{1} D_{1}-D_{1}^{2} \approx 0.25 \mathrm{~mm}^{2}$, leading to $2 r=1 \mathrm{~mm}<1.2 \mathrm{~mm}$, i.e., less than a maximum value allowed by the optical surface specification data. ${ }^{19}$ Respective quantity $d$ defined in Fig. 1 (a) is equal to $d \approx r^{2} /(2 R) \approx 0.83 \mu \mathrm{m}$. Then the flattening of a lens surface at the point of closest approach to the plate is $d-D_{1} \approx 0.33 \mu \mathrm{m}$ which is much less than $\Delta R$. 


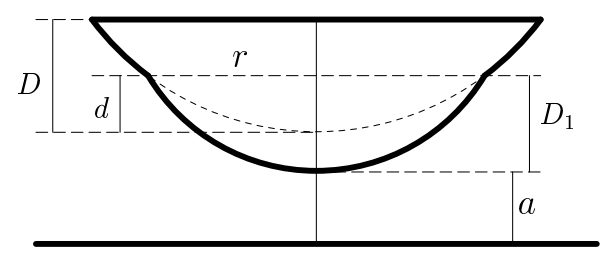

(a)

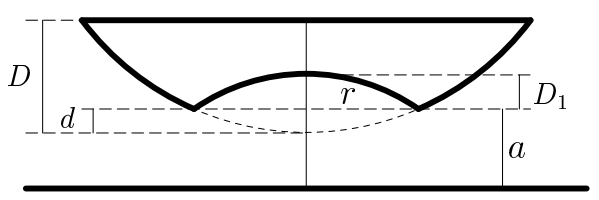

(b)

Fig. 2. The configuration of a spherical lens with curvature radius $R$ possessing a surface imperfection at the point of closest approach to a plate. (a) The bubble curvature radius is $R_{1}<R$. (b) The pit curvature radius is $R_{1}<R$. The relative sizes of the lens and imperfection are shown not to scale.

The general formulation of the PFA (4) should be applied taking into account that the surfaces of the bubble and of the lens are described by the equations

$$
z=R_{1}+a-\left(R_{1}^{2}-\rho^{2}\right)^{1 / 2}, \quad z=R+D_{1}-d+a-\left(R^{2}-\rho^{2}\right)^{1 / 2},
$$

respetively. Here $a$ is the distance between the bottom point of the bubble and the plate [see Fig. 1(a)]. Using (11) one arrives, instead of (6), at

$$
F_{s p}(a, T)=2 \pi \int_{a+D_{1}}^{a+D_{1}-d+D}\left(R-z+D_{1}-d+a\right) P(z, T) d z+2 \pi \int_{a}^{a+D_{1}}\left(R_{1}-z+a\right) P(z, T) d z .
$$

Now we take into consideration that the quantities $a, d$, and $D_{1}$ are smaller than the error in the determination of large radii $R$ and $R_{1}$. Then, calculating similar to (6) and (7), one finally obtains

$$
F_{s p}(a, T) \approx 2 \pi\left(R-R_{1}\right) \mathcal{F}_{p p}\left(a+D_{1}, T\right)+2 \pi R_{1} \mathcal{F}_{p p}(a, T) .
$$

We present a few computational results demonstrating that the Casimir force in Eq. (13) taking the flattening of a lens surface into account deviates significantly from the Casimir force $F_{s p}^{\text {perf }}$ in Eq. (8) obtained for perfect spherical surface. Computations of the quantity $F_{s p}(a, T) / F_{s p}^{\text {perf }}(a, T)$ were performed using (10) at $T=300 \mathrm{~K}$ within the separation region from 1 to $3 \mu \mathrm{m}$ [see the line labeled 1 in Fig. 1(b)]. As can be seen in Fig. 1(b), in the presence of a bubble leading to a flattening of lens surface shown in Fig. 1(a), the use of (8) for a perfect spherical surface instead of (13) considerably underestimates the magnitude of the Casimir force. Thus, at separations $a=1.0,2.0$, and $3.0 \mu \mathrm{m}$ the quantity $F_{s p} / F_{s p}^{\text {perf }}$ is equal to $1.458,1.287$, and 1.193 , respectively, i.e., the underestimation varies from $46 \%$ at $a=1 \mu \mathrm{m}$ to $19 \%$ at $a=3 \mu \mathrm{m}$.

Now we consider two more examples of surface imperfections, specifically, a bubble with the curvature radius $R_{1}=5 \mathrm{~cm}$ [see Fig. 2(a)] and a pit with the curvature radius $R_{1}=12 \mathrm{~cm}$ [see Fig. 2(b)]. In both cases the curvature radius of the lens remains the same $R=15 \mathrm{~cm}$. For the bubble we choose $D_{1}=1 \mu \mathrm{m}$ which results 
in $r \approx 0.32 \mathrm{~mm}, d \approx 0.33 \mu \mathrm{m}$, and $D_{1}-d \approx 0.67 \mu \mathrm{m}$ in agreement with allowed values. Equation (13) is evidently preserved with the new values of parameters. The computed values of the quantity $F_{s p}(a, T) / F_{s p}^{\text {perf }}(a, T)$ as a function of separation are shown by the line labeled 2 in Fig. 1(b). It can be seen that in this case the assumption of perfect sphericity of a lens surface considerably overestimates the magnitude of the Casimir force. Thus, at separations $a=1.0,2.0$, and $3.0 \mu \mathrm{m}$ the values of the quantity $F_{s p} / F_{s p}^{\text {perf }}$ are equal to $0.429,0.580$, and 0.689 , respectively, i.e., overestimation varies from $57 \%$ at $a=1 \mu \mathrm{m}$ to $36 \%$ at $a=3 \mu \mathrm{m}$.

Now we deal with a pit shown in Fig. 2(b). Here, the lens surface near the point of closest approach to the plate is concave up, i.e., in the direction of lens center. The related parameters are $D_{1}=1 \mu \mathrm{m}, r \approx 0.49 \mathrm{~mm}, d \approx 0.8 \mu \mathrm{m}$, and $d+D_{1} \approx 1.8 \mu \mathrm{m}$. The pit and lens surfaces are described by the equations

$$
z=a+D_{1}-R_{1}+\left(R_{1}^{2}-\rho^{2}\right)^{1 / 2}, \quad z=R+a-d-\left(R^{2}-\rho^{2}\right)^{1 / 2},
$$

respectively. Here, $a$ is the separation distance between the plate and points of a circle on the lens surface closest to it. Repeating calculations that have led to (13) with the help of (14), we obtain

$$
F_{s p}(a, T) \approx 2 \pi\left(R-R_{1}\right) \mathcal{F}_{p p}(a, T)+2 \pi R_{1} \mathcal{F}_{p p}\left(a+D_{1}, T\right)
$$

The computational results using (8), (10) and (15) are shown by the line labeled 3 in Fig. 1(b). Once again, the assumption of perfect lens sphericity significantly overestimates the magnitude of the Casimir force. Thus, at separations $a=1.0,2.0$, and $3.0 \mu \mathrm{m}$ the ratio $F_{s p} / F_{s p}^{\text {perf }}$ is equal to $0.314,0.496$, and 0.627 , respectively, i.e., overestimation varies from $69 \%$ at $a=1 \mu \mathrm{m}$ to $37 \%$ at $a=3 \mu \mathrm{m}$.

To conclude this section, we have shown that depending on the character of imperfections on the lens surface near the points of closest approach to the plate, the use of the PFA in the simplest form (8) can lead to either underestimated or overestimated Casimir force by a few tens of percent. Keeping in mind that the exact position of the point of closest approach on a spherical surface cannot be controlled with sufficient precision, it seems impossible to determine the character of surface imperfections near the point of closest approach microscopically for subsequent use of (13) or (15). This leads us to the conclusion that measurements of the Casimir force by using spherical lenses of centimeter-size curvature radii are fundamentally flawed in the sense that they can lead to unpredictable measurement results which cannot be reliably compared with theory.

\section{Further Objectionable Features}

\subsection{Whether or not the Casimir force is simply the retarded van der Waals force?}

The paper ${ }^{1}$ gives negative answer to this question because "the Casimir force does not depend on the properties of the individual atoms of the plates, but on their bulk properties". The same is, however, correct for the van der Waals force. It is common 
knowledge that the Lifshitz theory expresses both the van der Waals and Casimir forces between macrobodies in terms of dielectric permittivity of their materials which is a bulk property. The Lifshitz theory presents the unified description of both forces for dielectric and metallic plates. According to this theory, the van der Waals force occurs in the nonretarded limit. With the increase of separation after some transition regime, the van der Waals force transforms into the Casimir force in the retarded limit. The paper ${ }^{1}$ argues that "if the Casimir force was simply the retarded van der Waals force it would make little sense consider modifying the Casimir force, in a fundamental way, by altering the mode structure imposed by specially tailored boundary conditions". This argument, however, does not work because the nonretarded van der Waals force was just obtained ${ }^{33}$ from the mode structure determined by boundary conditions. Because of this, in the configuration of two material bodies separated with a gap it is beyond reason to make difference between the Casimir force and retarded van der Waals force.

\subsection{Is there conflict between the Casimir effect and electrical engineering?}

The paper ${ }^{1}$ applies classical Maxwell equations in combination with the plasma model at low frequencies and arrives at an effect that is not experimentally observed. Basing on this, it is concluded that "we are faced with discarding over a century of electrical engineering knowledge in order to explain a few $1 \%$ level Casimir force of questionable accuracy". This conclusion is, however, unjustified. Electrical engineering deals with real electromagnetic fields. It is a matter of common knowledge that classical Maxwell equations in the quasistatic limit lead to the Drude model dielectric permittivity which is inverse proportional to the frequency, and not to the plasma model which is only applicable in the range of infrared frequencies. This fact is underlined ${ }^{2,5,34}$ when the plasma model is used for the theoretical description of the Casimir force. The delicate point overlooked ${ }^{1}$ is that both classical electrodynamics and electrical engineering deal with real electromagnetic fields, whereas the Casimir effect deals with fluctuating electromagnetic fields possessing zero mean value. One of the postulates of quantum statistical physics that physical system reacts in the same way on real and fluctuating electromagnetic fields is presently under question from both theoretical and experimental sides. This does not touch any fact related to real electromagnetic fields. In view of this, it seems baseless to write about discarding over a centure of electrical engineering knowledge.

\section{Conclusions and Discussion}

In the foregoing we have drawn attention to a few facts relevant to the progress in measurements of the Casimir force. We have discussed what is incorrect in the argumentation $^{1}$ against precise experiments performed up to date and clarified some terminology of metrological character. 
The main new result of this paper is that all measurements of the Casimir force with centimeter-size spherical lenses are fundamentally flawed due to unavoidable deviations from a spherical shape arising from their manufacture. We have demonstrated that bubbles and pits satisfying constraints imposed by the optical surface specification data make inapplicable the simplified formulation of the PFA commonly used in the literature. We have also derived the expressions for the Casimir force applicable in the presence of bubbles or pits. It was shown that surface imperfections may lead to both a decrease and increase in the magnitude of the Casimir force up to a few tens of percent in the range of separations from 1 to $3 \mu \mathrm{m}$. Keeping in mind that experimentally it is impossible to determine the position of points of closest approach between interacting surfaces with sufficient precision, this in fact renders lenses of centimeter-size curvature radii impractical for measurements of the Casimir force. There might be additional problems in the application of spherical lenses of centimeter-size curvature radii for measuring the Casimir force which are not discussed here. We assert, however, that until the problem of deviations of lens surface from perfect spherical shape is somehow resolved any further measurements of the Casimir force using large spherical lenses are meaningless. Thus, the single remaining candidate for the registration of thermal effects in the Casimir force at micrometer separations is the configuration of two parallel plates.

The fundamental flaw discussed in this paper is not peculiar for spheres with much smaller radii used in numerous experiments performed by different authors by means of an atomic force microscope and micromachined oscillator (see review Ref. 2). For instance, surfaces of polystyrene spheres of about $100 \mu \mathrm{m}$ radii made from liquid phase preserve perfect spherical shape due to surface tension. The surface quality of such spheres was investigated using a scanning electron microscope and did not reveal any bubbles and scratches.

To conclude on a positive note, we agree with Ref. 1 that it is the reader who will finally decide which experiments in the Casimir force area are credible and which are not "based on verifiable facts and independent scientific analysis."

\section{Acknowledgments}

The authors are grateful to the Federal University of Paraíba (João Pessoa, Brazil) for kind hospitality. They were partially supported by CNPq (Brazil).

\section{References}

1. S. K. Lamoreaux, Progress in experimental measurements of surface-surface Casimir forces, in Casimir Physics, eds. D. Dalvit, P. Milonni, D. Roberts and F. da Rosa (Springer, Berlin, 2011); arXiv:1008.3640v1.

2. G. L. Klimchitskaya, U. Mohideen and V. M. Mostepanenko, Rev. Mod. Phys. 81, 1827 (2009).

3. R. S. Decca, D. López, E. Fischbach, G. L. Klimchitskaya, D. E. Krause and V. M. Mostepanenko, Phys. Rev. D 75, 077101 (2007); Eur. Phys. J. C51, 963 (2007).

4. B. W. Harris, F. Chen and U. Mohideen, Phys. Rev. A 62, 052109 (2000). 
5. M. Bordag, G. L. Klimchitskaya, U. Mohideen and V. M. Mostepanenko, Advances in the Casimir Effect (Oxford University Press, Oxford, 2009).

6. http://www.nist.gov/ts/msd/srm/index.cfm

7. S. Brandt, Statistical and Computational Methods in Data Analysis (North-Holland, Amsterdam, 1976).

8. R. S. Decca, D. López, E. Fischbach, G. L. Klimchitskaya, D. E. Krause and V. M. Mostepanenko, Ann. Phys. (N.Y.) 318, 37 (2005).

9. R. S. Decca and D. López, Int. J. Mod. Phys. A 24, 1748 (2009).

10. S. G. Rabinovich, Measurement Errors and Uncertainties. Theory and Practice (Springer-Verlag, New York, 2000).

11. D. A. R. Dalvit and R. Onofrio, Phys. Rev. D 80, 064025 (2009).

12. R. S. Decca, D. López, E. Fischbach, G. L. Klimchitskaya, D. E. Krause and V. M. Mostepanenko, Phys. Rev. D 79, 124021 (2009).

13. E. Fischbach, G. L. Klimchitskaya, D. E. Krause and V. M. Mostepanenko, Eur. Phys. J. C 68, 223 (2010).

14. R. Onofrio, Int. J. Mod. Phys. A 25, 2260 (2010).

15. W. J. Kim, M. Brown-Hayes, D. A. R. Dalvit, J. H. Brownell and R. Onofrio, Phys. Rev. A 78, 020101(R) (2008).

16. W. J. Kim, A. O. Sushkov, D. A. R. Dalvit and S. K. Lamoreaux, Phys. Rev. Lett. 103, 060401 (2009).

17. W. J. Kim, M. Brown-Hayes, D. A. R. Dalvit, J. H. Brownell and R. Onofrio, J. Phys.: Conf. Ser. 161, 012004 (2009).

18. R. S. Decca, D. López, E. Fischbach, G. L. Klimchitskaya, D. E. Krause, U. Mohideen and V. M. Mostepanenko, Phys. Rev. A 79, 026101 (2009).

19. http://www.prhoffman.com/technical/scratch-dig.htm

20. W. J. Kim, M. Brown-Hayes, D. A. R. Dalvit, J. H. Brownell and R. Onofrio, Phys. Rev. A 79, 026102 (2009).

21. R. S. Decca, E. Fischbach, G. L. Klimchitskaya, D. E. Krause, D. López, U. Mohideen and V. M. Mostepanenko, arXiv:0904.4702; Int. J. Mod. Phys. A, this issue (2011).

22. Q. Wei, D. A. R. Dalvit, F. C. Lombardo, F. D. Mazzitelli and R. Onofrio, Phys. Rev. A 81, 052115 (2010).

23. F. Chen, U. Mohideen, G. L. Klimchitskaya and V. M. Mostepanenko, Phys. Rev. A 74, 022103 (2006).

24. F. Chen, G. L. Klimchitskaya, V. M. Mostepanenko and U. Mohideen, Phys. Rev. Lett. 97, 170402 (2006).

25. H.-C. Chiu, G. L. Klimchitskaya, V. N. Marachevsky, V. M. Mostepanenko and U. Mohideen, Phys. Rev. B 80, 121402(R) (2009); ibid 81, 115417 (2010).

26. V. B. Bezerra, G. L. Klimchitskaya, U. Mohideen, V. M. Mostepanenko and C. Romero, Phys. Rev. B 83, 075417 (2011).

27. B. V. Derjaguin, Kolloid. Z. 69, 155 (1934).

28. J. Błocki, J. Randrup, W. J. Swiatecki and C. F. Tsang, Ann. Phys. (N.Y.) 105, 427 (1977).

29. B. Geyer, G. L. Klimchitskaya and V. M. Mostepanenko, Phys. Rev. A 82, 032513 (2010).

30. K. A. Milton, The Casimir Effect (World Scientific, Singapore, 2001).

31. S. K. Lamoreaux, Phys. Rev. Lett. 78, 5 (1997); 81, 5475(E) (1998).

32. A. O. Sushkov, W. J. Kim, D. A. R. Dalvit and S. K. Lamoreaux, Nature Phys. 7, 230 (2011).

33. N. G. van Kampen, B. R. A. Nijboer and K. Schram, Phys. Lett. A 26, 307 (1968).

34. V. M. Mostepanenko and G. L. Klimchitskaya, Int. J. Mod. Phys. A 25, 2302 (2010). 